\title{
BILLION-CYCLE ULV ELECTROSTATIC RF MEMS SWITCH
}

\author{
Tsung-Kuan A. Chou, Hanan Bar, John Heck, Qing Ma, Joseph B. Melki, Quan Tran, Shlomy Tubul, Boaz \\ Weinfeld, Nataly Ziharev \\ Intel Corporation \\ SC9-09, 2200 Mission College Blvd., Santa Clara, CA 95052, USA.
}

\begin{abstract}
An ultra-low-voltage (ULV) electrostatic RF MEMS switch has been demonstrated with actuation voltage as low as $\sim 1.5 \mathrm{~V}$, the lowest reported. The switch is designed to collapse on to actuation electrode with zipping action. The device consists of multiple spring constants, which maximizes the open force while reducing the pull-in voltage to $<3 \mathrm{~V}$. Physical stopper design eliminates the usage of dielectric between electrodes so that actuation charging problem is minimized. The device is fabricated using a low-stressgradient polysilicon as beam structure to realize ULV actuation. The ULV switch has achieved cold-switching lifetime of $\sim 1 \times 10^{9}$ cycles with close speed of $\sim 50 \mu$ s at $3.2 \mathrm{~V}$ and open speed of $\sim 25 \mu \mathrm{s}$.
\end{abstract}

\section{INTRODUCTION}

The RF MEMS switch provides disruptive technology to wireless communications due to its high linearity and low insertion loss. Resistive contact switch is most suitable for frequency in the range of 0 to $6 \mathrm{GHz}$. Different actuation approaches have been used in contact switches, such as electrostatic, electromagnetic, and thermal actuations [1]-[4]. Electrostatic RF MEMS switch is the best candidate for its low powder consumption, and integration compatibility with LC filters and CMOS circuitries. Fig.1 illustrates one of the conventional RF MEMS contact switches. It consists of a thick metal cantilever beam with a bottom actuation electrode. A contact tip is located at front end of the cantilever, corresponding to a RF trace underneath. The pull-in voltage of the switch can be expressed in Eq. 1, where $\mathrm{k}_{1}$ is the cantilever spring constant, $\mathrm{g}$ is the gap between electrodes, and $\mathrm{A}$ is the actuation area. When a DC voltage of $>\mathrm{Vp}$ is applied on the actuation electrode, the top beam is pulled down electro-statically. The cantilever tip makes contact on bottom RF trace so that electric signal can pass through. When actuation voltage is removed, the

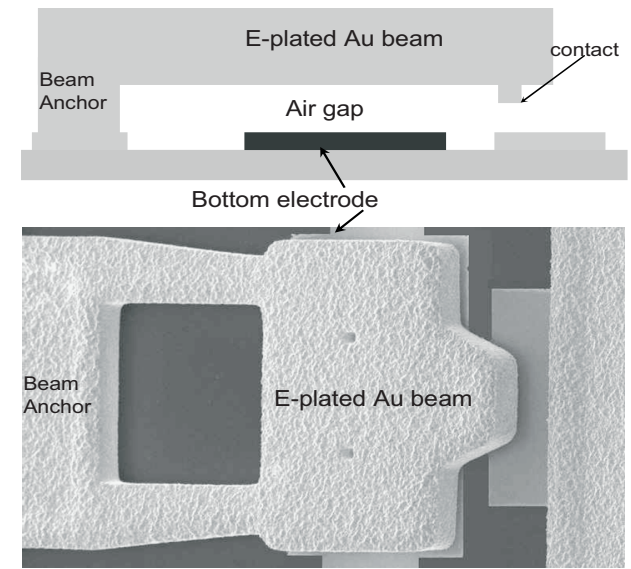

Figure 1. Schematic of conventional electrostatic contact switch with electroplated gold cantilever beam and SEM of a fabricated 40V RF MEMS switch. cantilever's restoring force opens the switch contact.

$$
V p=\sqrt{\frac{8 k_{1} g^{3}}{27 \varepsilon_{o} A}}
$$

A 40V RF MEMS switch was fabricated as shown in Fig 1. It has achieved insertion loss of $<0.3 \mathrm{~dB}$, isolation of $\sim 32 \mathrm{~dB}$ at $2 \mathrm{GHz}$, and lifetime of $>300 \times 10^{6}$ cycles. Similar switch with lifetime of $>1 \times 10^{9}$ cycles is also reported by other researchers with actuation voltage of $>80 \mathrm{~V}$ [5]. However, such switches require an expensive high-voltage (HV) driver, which can cost significantly more than the switch itself. As a result, there is a strong demand on the development of ULV MEMS switch which can be actuated at 3.3V. Electro-statically actuated MEMS switch with actuation voltage of $6 \mathrm{~V}$ has been reported [6]. However, the switch has very limited lifetime obtained. Meanwhile, switch with lifetime of $>100 \mathrm{M}$ cycles and actuation voltage of $<5 \mathrm{~V}$ have not been demonstrated to-date. This research work attempts to develop an electrostatic contact switch with actuation voltage of $<3.3 \mathrm{~V}$ and lifetime of $\sim 1 \times 10^{9}$ cycles.

\section{DESIGN OF ULV MEMS SWITCH}

In order to achieve ultra low pull-in voltage, one needs to either reduce spring constant $\mathrm{k}_{1}$, reduce the gap $\mathrm{g}$ between electrodes, or increase the actuation area A. Most favorable approach is to reduce the air gap and increase the actuation area. Some reduction on the spring constant is also necessary in order to reach $3 \mathrm{~V}$ actuation. However, the beam's curving amount from stress gradient increases drastically when the cantilever or electrode area increases. The final air gap between electrodes is dominated by the stress gradient curving, which prevents Vp reduction. Peroulis et. al. have achieved $6 \mathrm{~V}$ actuation voltage by making the structure highly compliant. The cantilever spring constant $\mathrm{k}_{1}$ has been reduced down to $\sim 0.5 \mathrm{~N} / \mathrm{m}$ with serpentine clamp-beam structure. However, since the cantilever's restoring force is also used to open the switch, such ultra compliant device can easily suffer from contact stiction failure with open force of only about $1 \mu \mathrm{N}$.

Figure 2 illustrates the top view (Fig.2a) and crosssection (Fig.2b) view of zipper polysilicon switch structure with back contact design. The top beam is composed by a low stress gradient (LSG) polysilicon. This polysilicon cantilever serves as the main switch actuation structure, and carries the metal conductor to open and close the switch contact for RF signal transmission. LSG poly silicon is used for its material robustness. Meanwhile, the consistent stress control of the polysilicon film also makes small air gap feasible for ultra low voltage actuation. The polysilicon beam consists of two parts, short polysilicon arms with stiff spring constant $\mathrm{k}_{2}$ and a large electrode plate attached to the stiff arms. A bottom 
2a) Top view schematic of ULV poly switch



2b) Cross-section view of zipper poly switch

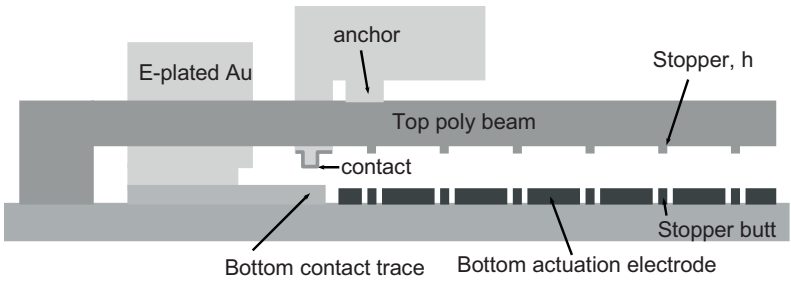

2c) Pull-in of top beam with ultra low $\mathrm{Vp}$

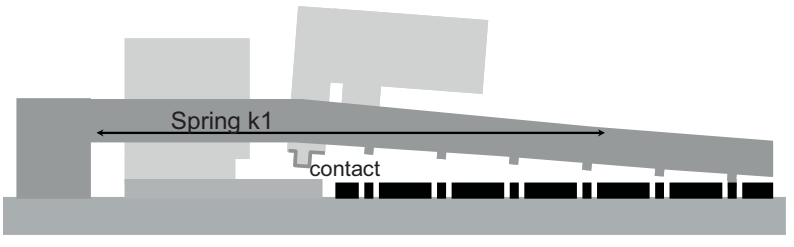

2d) Collapsing of top beam with zipping action and contact made

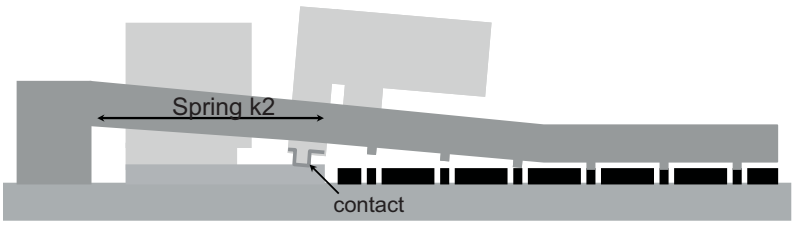

Figure 2. Design and operation of ULV collapsing zipper switch.

electrode is located directly underneath the top electrode. This actuation electrode will experience a much longer cantilever beam and much more compliant spring constant $\mathrm{k}_{1}$. The bottom side of LSG beam contains many stopper bumps. Meanwhile, there are electrically isolated butts distributed within actuation electrode in corresponding to the stopper bumps above. These stopper pairs are functioning as insulator layer between two electrodes, which allow the top beam collapsing onto bottom electrode without suffering from any electrical short. Since no actual dielectric layer is used, it significantly reduces actuation charging problem as commonly observed in uni-polar electrostatic actuation [7].

The switch RF contact metal is located on the LSG polysilicon close to cantilever anchor in corresponding to the short arms with spring constant $\mathrm{k}_{2}$. The thick metal provides low trace resistance for RF signal transmission and the robust poly beam structure provides ultra low voltage switch actuation. Such design configuration has separated the very compliant spring $\mathrm{k}_{1}$ for ultra low $\mathrm{Vp}$ and stiff $\mathrm{k}_{2}$ for large open force. The ULV switch open force at contact is expressed in Eq. 2, where $\Delta z$ is the contact tip displacement.

$$
F_{\text {open }}=k_{2} \Delta z
$$

When DC voltage is applied to the actuation electrode, top beam collapses toward the actuation electrode with spring $\mathrm{k}_{1}$ as shown in Fig.2c. Pull-in voltage is determined by $\mathrm{k}_{1}$, air gap and the electrode dimension as depicted in Eq. 1. The spring $\mathrm{k}_{1}$ is in the range of $10 \mathrm{~N} / \mathrm{m}$ based on the cantilever and electrode dimension design. $\mathrm{Vp}$ is expected to be $<3 \mathrm{~V}$ for air gap of $<1 \mu \mathrm{m}$. When the top electrode collapses, electrostatic force increases drastically due to very small gap between electrodes. The electrostatic force depends on the stopper height design (40nm to $100 \mathrm{~nm})$. Top beam continues collapsing with zipping action towards the beam anchor until contact is made as shown in Fig.2d. Meanwhile, the cantilever restoring force increases with the zipping action until the stiff spring $\mathrm{k}_{2}(100 \mathrm{~N} / \mathrm{m}$ to $250 \mathrm{~N} / \mathrm{m})$ is reached. Larger open force with spring $\mathrm{k}_{2}$ can reduce contact stiction failure when actuation is removed. Spring force of $>10 \mu \mathrm{N}$ is required to overcome the contact stiction based on nano-indenter and actual switch contact tests with contact force of $50 \mu \mathrm{N}$ to $100 \mu \mathrm{N}$. Contact and open forces are optimized in the range of $30 \mu \mathrm{N}$ to $50 \mu \mathrm{N}$ for $3.3 \mathrm{~V}$ in the design. The zipping action design in this structure reduces the large impact force directly on contact. Less contact bouncing, deformation, and stiction is expected. Meanwhile, the switch opens with zipping action, which reduces the stiction on electrodes as well. Note that this initial ULV switch development is focusing on the switch's functionality and electro-mechanical responses. RF performance was not optimized in this work.

\section{FABRICATION OF ULV LOW STRESS GRADIENT POLYSILICON SWITCH}

A low stress gradient LPCVD polysilicon film has been developed. The surface profile of polysilicon cantilevers at open state is shown in Fig 3. The stress-uncompensated polysilicon film shows a large internal stress gradient with $>9 \mu \mathrm{m}$ tip displacement on $350 \mu \mathrm{m}$ long beam as seen in Fig 3a. Despite the large stress gradient, these polysilicon beams have consistent stress bending across wafer. After deposition process tuning and stress compensation, low stress gradient polysilicon was obtained as shown in Fig. 3b. These LSG poly beams showed reproducible stress gradient bending of $\sim 50 \mathrm{~nm}$ over $350 \mu \mathrm{m}$ beam with deviation of $\sim 22 \mathrm{~nm}$, significantly less than the electroplated Au beam.

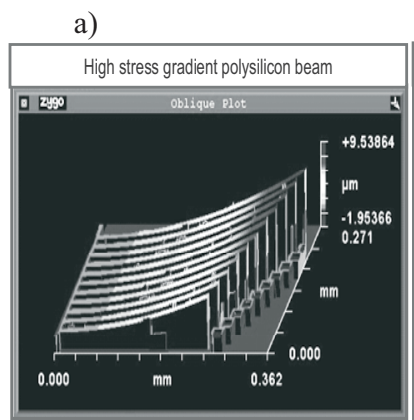

b)

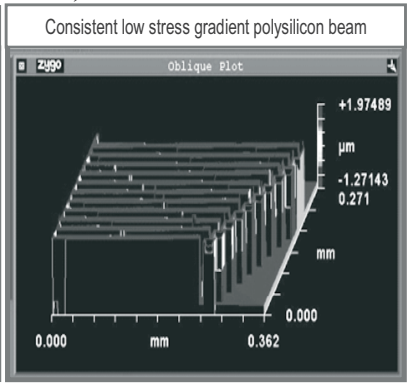

Figure 3. a) Uniform high stress gradient polysilicon with $\Delta z>9 \mu m$ on $350 \mu m$ poly beam. b) Consistent low stress gradient poly silicon with $\triangle z \sim 0.05 \mu \mathrm{m}$ on $350 \mu \mathrm{m}$ beam for ULV switch. 
a)

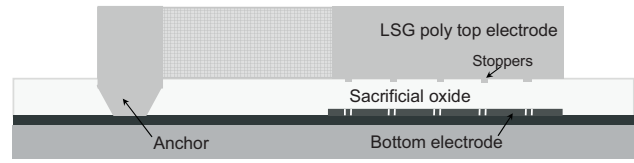

b)

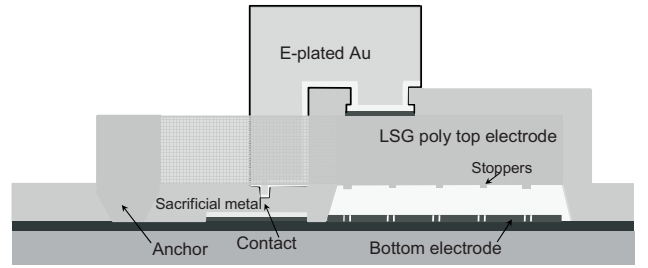

c)



Figure 4. Process illustration of ULV polysilicon switch

The basic process of ULV polysilicion switch starts with isolation nitride on high-resistivity silicon substrate $(>5 \mathrm{k} \Omega-\mathrm{cm})$. A thin polysilicon bottom electrode $(0.1 \mu \mathrm{m} \sim 0.2 \mu \mathrm{m})$ was deposited and patterned to form the actuation pad and resistor. LPCVD sacrificial oxide was deposited to define the air gap. Polysilicon stopper bump formation and anchor via etch were performed and followed by the deposition of LSG polysilicon $(1.5 \mu \mathrm{m} \sim 2.5 \mu \mathrm{m})$. Polysilicon beam and top electrode were then pattern as seen in Fig 4a. Bottom contact metal were then deposited and patterned. Sacrificial material was then deposited to define the gap between bottom metal and top contact. Anchor etch was then performed and the contact metal was deposited followed by thick gold layer

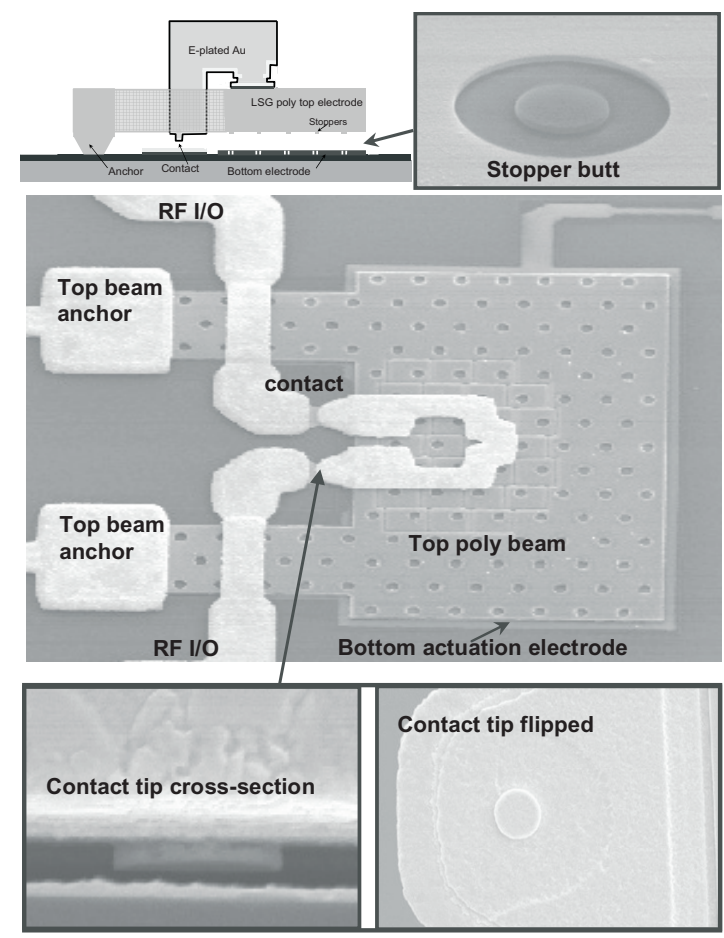

Figure 5. SEM views of fabricated ULV polysilicon switch. electroplating to construct the switch RF I/Os as shown in Fig. 4b. The sacrificial layers were then etched away. And the device was finally released by supercritical $\mathrm{CO}_{2}$ drying method to prevent "stiction" (Fig. 4c).

Fig. 5 shows the fabricated ULV switch with two contacts in series. SEMs of the stopper and contact are also illustrated. The RF $\mathrm{I} / \mathrm{O}$ traces reach to the switch contact with air bridges over the cantilever arms as seen in the photo. This approach provides more flexibility in the routing. However, one should take into account the capacitive coupling issue while design such type of air bridge. $\mathrm{RF}$ optimization is needed but is beyond the scope of this paper focus.

\section{DETAILED TEST RESULTS}

The fabricated ULV polysilicon switches were first screened for $\mathrm{Vp}$ uniformity. Figure 6 shows the wafer-level uniformity of the pull-in voltage obtained from laser scanning vibrometer (LSV) measurement. LSV in-situ monitored the cantilever mechanical closing velocity and captured both the applied voltage at the moment when the top beam collapsed. The fabricated switches have an average $\mathrm{Vp}$ of $\sim 2.5 \mathrm{~V}$, higher than expected of $1.5 \mathrm{~V}$ to $2 \mathrm{~V}$. Functional switch with lowest $\mathrm{Vp}$ of $\sim 1.5 \mathrm{~V}$ has been demonstrated, the lowest voltage for electrostatic switch actuation to author's knowledge. The variation of $\mathrm{Vp}$ was due to the stress mismatch of the electroplated gold anchoring on the polysilicon beam. It resulted in slight upward bending of the polysilicon beam. Due to variation of the gold stress, the gap variation between ULV switches leads to Vp differences across the wafer.

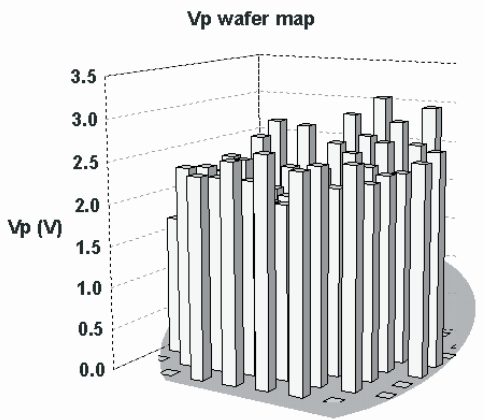

Figure 6. Wafer-lever uniformity of pull-in voltage.

The switching speed of the ULV switch was evaluated by applying a prompt $-3.2 \mathrm{~V}$ at actuation electrode. Hot switching measurement was used to obtained the close and open speed, $t_{o n}$ and $t_{\text {off. }}$ Figure $7 \mathrm{a}$ illustrates the schematic of test setup for hot switching. Oscilloscope was used to capture the actuation voltage and the signal through switch contact simultaneously. A $500 \mathrm{kHz}$ AC signal was imposed on one of the switch RF trace I/Os while the oscilloscope monitored the signal on the other RF I/O with $50 \Omega$ load. When switch was closed by the applied voltage $(\sim-$ $3.2 \mathrm{~V}$ ), switch contact was made and formed a conduction loop. The AC signal then passed through the contacts and was detected by the OSC. It can be seen from Fig. $7 b$ that the switch has close speed $t_{o n}$ of $\sim 50 \mu \mathrm{s}$. No contact bounce was observed in the measurements. When the actuation voltage was removed $(0 \mathrm{~V})$, the switch broke the contact. AC signal was not able to pass onto the $50 \Omega$ load terminal after $\sim 25 \mu \mathrm{s}$, corresponding to the switch open speed $t_{\text {off }}$.

The ULV switch has achieved contact resistance of $\mathrm{Rc}<=1 \Omega /$ contact. Each switch contains two contacts in series and 
$2 \Omega$ trace resistance in design, resulting in total trace resistance of $\sim 4 \Omega$. Although the ULV switch design was not optimized for RF performance, it exhibited $<0.4 \mathrm{~dB}$ insertion loss up to $6 \mathrm{GHz}$. The device isolation is $\sim-20 \mathrm{~dB}$ at $2 \mathrm{GHz}$. The current RF trace structure has known capacitive coupling between RF I/Os, which can be improved by design modification. A single-contact ULV polysilicon is also developed to future improve. It was observed that the contact cleanliness is crucial to ULV switch for its relatively low contact force $(25 \mu \mathrm{N} \sim 50 \mu \mathrm{N})$. Clean contact surface is the key to reliable switch operation. Contact failure due to drastic Rc increase often occurred much faster if the contact was not clean (higher initial Rc). An improved switch cleaning process was used to achieve low initial contact resistance. However, as the fabrication process improves to cleaner surface, the probability of contact stiction increases as well. Experiment result showed that $\mathrm{Au}$ contact can suffer from contact stiction within $100 \times 10^{6}$ cycles even without RF signal passing through the contact. It is suspected that the contact tip has deformed during cycling and contact stiction force becomes too high to be overcome by the switch's open force $(<=50 \mu \mathrm{N})$. Hard metals showed less contact deformation and stiction after persistent contact impact cycling.

a)

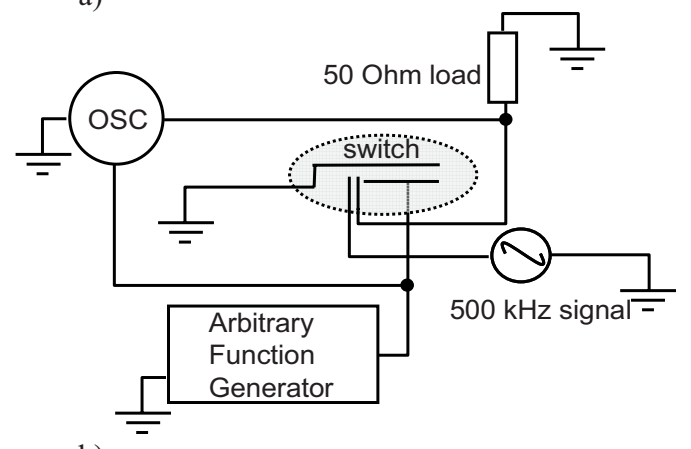

b)



Figure 7. Measured switching speed of ULV MEMS switch. $t_{\text {on }} \sim 50 \mu \mathrm{s}$ and $t_{\text {off }} \sim 25 \mu \mathrm{s}$.

The ULV polysilicon switch is intended for reconfigurable $\mathrm{RF}$ receiver in this work. The RF signal power strength is expected to be less than $0 \mathrm{dBm}$. Test result showed that the main switch failure was due to increase of contact resistance at such power level. Preliminary analysis indicated that there was organic built up at contact surface. Higher input power $(>0 \mathrm{dBm})$ could breakdown this layer and prolong the switch's low contact resistance. However, to mimic the low RF power in reconfigurable receiver, the ULV switch was cold-switched with input powder of $-10 \mathrm{dBm}$ to $0 \mathrm{dBm}$ range. Figure 8 shows the cold-switching lifetime of the fabricated ULV switch. The trace resistance $(\sim 2 \Omega)$

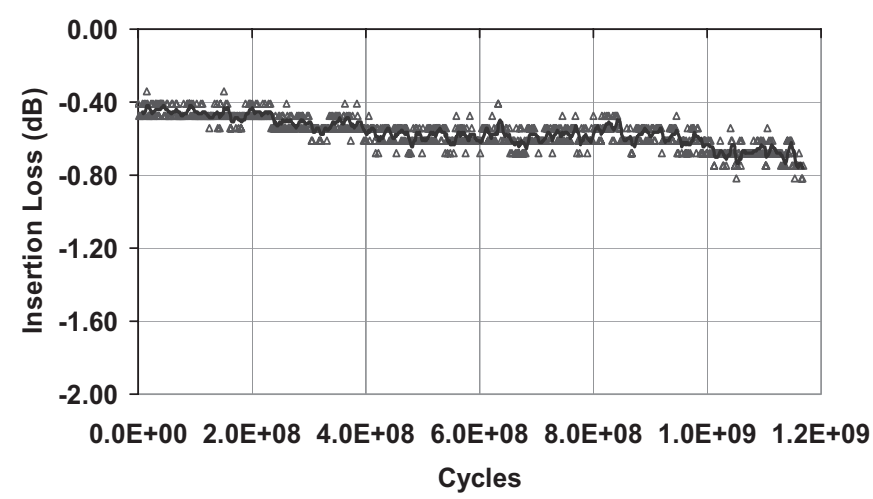

Figure 8. Cold-switching lifetime of ULV switch. Insertion loss includes $2 \Omega$ trace resistance.

is included in the insertion loss result. The switch reached $\sim 1.15 \times 10^{9}$ cycles before drastic increase on the insertion loss. The device switching lifetime was strongly affected by the surface and environment cleanliness as discussed previously. Hermetic encapsulation is necessary to ensure reliable switch lifetime.

\section{CONCLUSIONS}

A 3.3V ultra low voltage electrostatic MEMS switch has been developed. The device structure design separates the spring constants for $\mathrm{Vp}$ and open force. Contact resistance of $<1 \Omega /$ contact has been achieved with improved process cleanliness. The ULV switch demonstrated cold-switching lifetime of $>1 \mathrm{~B}$ cycles with close speed of $\sim 50 \mu$ s and open speed of $\sim 25 \mu \mathrm{s}$. The development results suggest that ultra-low-voltage electrostatic switch with $3.3 \mathrm{~V}$ can be realized without using HV driver.

\section{ACKNOWLEGEMENT}

The authors wish to thank all the contributing members, TMG, WNG, and NBI at Intel Corporation for the support of this work.

\section{REFERENCES}

[1] R. Mihailovich, et. al., "MEMS Relay for Reconfigurable RF Circuits," IEEE Microwave Comp. Lett., Vol. 11, No.2, Feb. 2001, pp. 53-55.

[2] P. Zavracky, et. al., "Microswitches and Microrelays with a View toward Microwave Applications," Int. J. RF Microwave CAE, Vol. 9, No. 4, 1999, pp. 338-347.

[3] P. Blondy, et. al., " Packaged mm-Wave Thermal MEMS Switches," $31^{\text {st }}$ European Microwave Conference, Vol. 1, Sep. 2001, pp. 283-286.

[4] M. Ruan, et. al., " Latching Micromagnetic Relays," IEEE J. Microelectromech. Systems, Vol. 10, Dec. 2001, pp. 511-517.

[5] S. Majumder, et.al., "A Packaged, High-Lifetime Ohmic MEMS RF Switch," IEEE MTT-S Intl. Microwave Symp. Dig., June 2003, pp. 1935-1938.

[6] D. Peroulis, et. al., "Electromechanical Considerations in Developing Low-Voltage RF MEMS Switches," IEEE Trans. Microwave Theory Tech., Vol. 51, No. 1, Jan. 2003, pp 259270.

[7] X. Yuan, et. al., "Modeling and Characterization of DielectricCharging Effects in RF MEMS Capacitive Switches," IEEE MTT-S Intl. Microwave Symp. Dig., June 2005, pp 753-756. 